\title{
Influência do teor de argila nas propriedades do polipropileno e a reciclagem dos nanocompósitos obtidos por injeção
}

\author{
Influence of nanoclay content on polypropylene \\ properties and recycling of nanocomposites \\ obtained by injection
}

Guilherme Chini Colonetti ${ }^{1}$, Jeniffer Katarine Wanzeler Fuckner ${ }^{2}$, André Lourenço Nogueira ${ }^{3}$, Ana Paula Testa Pezzin ${ }^{4}$

\begin{abstract}
1,2,3,4 Universidade da Região de Joinville - UNIVILLE - Mestrado em Engenharia de Processos, Rua Paulo Malschitzki 10. Zona Industrial.CEP:89.201-974, Joinville-SC, Brasil - 89219-710, Joinville, SC, e-mail: anapezzin@yahoo.com.br
\end{abstract}

\begin{abstract}
RESUMO
Neste trabalho foram preparados nanocompósitos de polipropileno e montmorilonita organofílica PP/MMTO, nas concentrações de 1, 2, 5 e 7\% pela técnica de intercalação fundida diretamente em uma injetora hidráulica monorosca. O material obtido foi processado, obtendo corpos de prova chamados de primários em formato de gravata. O reprocessamento foi realizado com a moagem do material em um moinho de facas. Logo após, o material moído foi injetado novamente obtendo o segundo ciclo do material e, posteriormente, até atingir o quarto ciclo. As amostras foram avaliadas por microscopia eletrônica de varredura (MEV), ensaio mecânico sob tração, análise termogravimétrica (TGA) e calorimetria exploratória diferencial (DSC). Os resultados de resistência à tração e módulo de Young para as formulações contendo nanoargila não apresentaram alterações significativas, fazendo com que a nanoargila atuasse como carga. As análises de TGA mostraram que quando adicionado 2\% MMTO, a temperatura de degradação ( $\mathrm{T}_{\text {onset }}$ ) aumentou 17,10\% quando comparada com o PP, sendo esse o melhor resultado encontrado. As curvas de DSC não mostraram variação na temperatura de fusão $\left(\mathrm{T}_{\mathrm{m} 2}\right)$ para as formulações contendo MMTO e também não para as formulações reprocessadas. Já o grau de cristalização $(\alpha c)$ apresentou variações significativas, tendo como destaque a formulação com $5 \%$ de MMTO, onde o $\alpha$ c foi $66,70 \%$, cerca de $26,80 \%$ superior ao polipropileno.
\end{abstract}

Palavras-chave: injeção, montmorilonita organofílica, nanocompósitos, polipropileno, reciclagem.

\section{ABSTRACT}

In this work, polypropylene and organophilic montmorillonite PP/MMTO nanocomposites were prepared at the concentrations of $1,2,5$ and $7 \%$ were prepared through the melt intercalation by directly at the single screw hydraulic injector. The obtained material was processed, obtaining specimens called ties in the form of ties. Reprocessing was performed by milling the material in a knife mill. Soon after the ground material was injected again obtaining the second cycle of the material and, later, until reaching the fourth cycle. The samples were evaluated by scanning electron microscopy (SEM), mechanical tensile test, thermogravimetric analysis (TGA) and differential scanning calorimetry (DSC). The results of tensile strength and Young's modulus for the nanoclay containing formulations did not show significant changes, causing the nanoclay to act only as a charge. The TGA analysis showed that when $2 \%$ MMTO was added, the degradation temperature (Tonset) increased $17.10 \%$ when compared to PP, which was the best result found. The DSC curves did not show variation in the melt temperature (Tm2) for the formulations containing MMTO and also for the reprocessed formulations. On the other hand, the degree of crystallization $(\alpha \mathrm{c})$ showed significant variations, with emphasis on the formulation with 5\% MMTO, where $\alpha \mathrm{c}$ was $66.70 \%$, about $26.80 \%$ higher than PP.

Keywords: Injection, nanocomposites, organophilic montmorillonite, polypropylene, recycling.

\section{INTRODUÇÃO}


O polipropileno (PP) é um termoplástico semicristalino pertencente ao grupo das poliolefinas, sendo um dos polímeros comercialmente mais importantes pela sua aplicabilidade, viabilidade econômica e durabilidade ${ }^{[1]}$. O campo de aplicação dos polímeros tem sido largamente ampliado, onde novas aplicações requerem, necessariamente, novas propriedades que muitas vezes o polímero puro não possui.

Neste contexto, surgem os nanocompósitos poliméricos, como uma das formas de modificar as propriedades dos polímeros por meio da incorporação de nanoreforços ${ }^{[2]}$. Um dos materiais nanométricos utilizados em pesquisas com nanocompósitos e suas aplicações são as argilas, devido à variedade e às interessantes propriedades que esses materiais apresentam, sendo alguma delas o aumento das propriedades mecânicas, aumento na estabilidade térmica, baixa permeabilidade a gases, água, elevada resistência química e retardância de chamas. No entanto, várias dessas aplicações só são possíveis após a organofilização, permitindo ampliar as faixas de aplicação das nanoargilas, gerando novos materiais e novas aplicações ${ }^{[3]}$.

A montmorilonita é um dos filosilicatos mais usados na obtenção de nanoargilas organofílicas, possuindo duas características que a torna mais interessante para a aplicação em nanocompósitos: a capacidade de as camadas lamelares incharem ao se separarem uma das outras e a capacidade de troca de cátion, onde são comumente modificadas com sais de amônio quaternário para favorecer a mistura polímero/nanoargila. Mesmo assim, para melhorar ainda mais as propriedades e a intercalação e adesão do polímero com a nanoargila organofílica é também utilizado um agente compatibilizante ${ }^{[4]}$.

Os agentes compatibilizantes polares promovem melhor adesão entre a matriz e as partículas, auxiliando também na dispersão das partículas no polímero. O PP graftizado com anidrido maleico é o compatibilizante mais utilizado comercialmente em pesquisas ${ }^{[5]}$. Estudos no processo de reciclagem de polímeros vêm sendo realizados em várias Universidades, Institutos de Pesquisas e empresas do mundo, constatando que o polímero processado uma vez pode apresentar alterações em suas propriedades, causadas por mudanças químicas, tais como a degradação térmica e a oxidação devido ao ciclo de aquecimento.

A forma mais comum para a reciclagem dos plásticos é a reciclagem mecânica, que consiste na reutilização de resíduos para obtenção de outro artefato plástico, por meio de reprocessamento em equipamentos industriais de transformação de plásticos ${ }^{[6]}$. Neste contexto, o presente estudo buscou obter nanocompósitos de PP com diferentes percentuais de MMTO, utilizando o PP graftizado com anidrido maleico como agente compatibilizante, através do processamento por injeção, obtendo corpos de prova denominados de primários e com até quatro ciclos de reprocessamento.

O estudo teve como objetivo geral avaliar a influência do teor de nanoargila nas propriedades morfológicas, mecânicas e térmicas dos nanocompósitos de PP/MMTO, bem como avaliar a influência da reciclagem nas propriedades desses compósitos obtidos por injeção. Para atender o objetivo proposto, fez-se necessário atingir alguns objetivos específicos, como: obter nanocompósitos de PP/MMTO pelo processo de injeção, caracterizar as formulações primárias e reprocessadas por microscopia eletrônica de varredura (MEV), ensaio mecânico sob tração, análise termogravimétrica (TGA) e calorimetria exploratória diferencial (DSC) e avaliar e comparar a influência do teor de nanoargila $(1,2,5$ e 7\%) nas propriedades mecânicas, térmicas e morfológicas dos nanocompósitos de PP/MMTO com relação ao PP.

\section{MATERIAIS E MÉTODOS}

Os materiais utilizados para a preparação dos nanocompósitos foram o polipropileno do tipo $35 \mathrm{H} 35$ da marca PROPILCO, o agente compatibilizante polipropileno graftizado com anidrido maleico (PP-g-AM) da marca

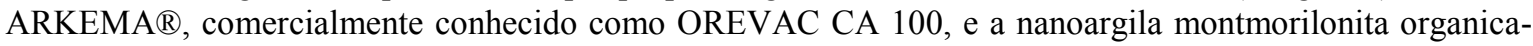
mente modificada com sais quaternários de amônio da marca SPECTROCHEM ${ }^{\circledR}$, nome comercial Surftreat $\mathrm{RM}-13479$.

Os nanocompósitos foram processados em uma injetora hidráulica da marca Haitan, modelo HTF90W/J5 monorosca, com perfil de temperaturas nas respectivas zonas de aquecimento de: $28^{\circ} \mathrm{C}, 211^{\circ} \mathrm{C}$, $216{ }^{\circ} \mathrm{C}, 200{ }^{\circ} \mathrm{C}, 184{ }^{\circ} \mathrm{C}$. Para o reprocessamento das amostras, estas foram moídas em um moinho da marca RONE $®$ da linha $\mathrm{W}$, modelo $210 / 3$ com $400 \mathrm{rpm}$. Primeiramente, prepararam-se as seis formulações primárias, mantendo o percentual de PP-g-AM em 15\%, alterando as concentrações de nanoargila (MMTO) em 1, 2,5 e $7 \%$ e complementando com PP até atingir $100 \%$ da formulação. A massa total dessas formulações foi de $1,5 \mathrm{~kg}$. A homogeneização das formulações primárias foi realizada manualmente, onde agitou-se por aproximadamente cinco minutos. Logo após a homogeneização, cada formulação primária foi diretamente processada em uma injetora. Os corpos de prova das formulações primárias foram gerados no formato gravata com dimensões de 162,40 × 18,9 x 13,1 x 3,20mm, valores estes dentro dos parâmetros estabelecidos pela norma ASTM D638 ${ }^{[7]}$. Para o reprocessamento dos corpos de prova primários, os mesmos foram moídos, gerando novas amostras cuja sua nomenclatura recebeu identificações com os sufixos $1 \mathrm{R}$ e $4 \mathrm{R}$ para indicar o 
número de ciclos de reprocessamento, sendo $1 \mathrm{R}$ um ciclo e $4 \mathrm{R}$ quatro ciclos de reprocessamento. Todas as formulações utilizadas neste trabalho estão apresentadas na Tabela 1. Vale ressaltar que para o reprocessamento das amostras, o material foi moído e injetado novamente com os mesmos parâmetros de injeção utilizados nos ciclos anteriores.

Tabela 1: Formulações elaboradas com suas nomenclaturas e números de ciclos

\begin{tabular}{|l|l|l|l|l|l|}
\hline $\mathbf{N}^{\circ}$ & PP PURO (\%) & PP-G-AM (\%) & NANOARGILA (\%) & NOMECLATURA & $\mathbf{N}^{\circ}$ DE CICLOS \\
\hline 1 & 100 & & & PP & Primária \\
\hline 2 & 100 & & & PP.1R & 1 \\
\hline 3 & 100 & & & PP.4R & 4 \\
\hline 4 & 84 & 15 & 1 & PP.AM.1\%MMTO & Primária \\
\hline 5 & 84 & 15 & 1 & PP.AM.1\%MMTO.1R & 1 \\
\hline 6 & 84 & 15 & 1 & PP.AM.1\%MMTO.4R & 4 \\
\hline 7 & 83 & 15 & 2 & PP.AM.2\%MMTO & Primária \\
\hline 8 & 83 & 15 & 2 & PP.AM.2\%MMTO.1R & $\mathrm{X}^{*}$ \\
\hline 9 & 83 & 15 & 2 & PP.AM.2\%MMTO.4R & $\mathrm{X}^{*}$ \\
\hline 10 & 80 & 15 & 5 & PP.AM.5\%MMTO & Primária \\
\hline 11 & 80 & 15 & 5 & PP.AM.5\%MMTO.1R & 1 \\
\hline 12 & 80 & 15 & 5 & PP.AM.5\%MMTO.4R & $\mathrm{X}^{*}$ \\
\hline 13 & 78 & 15 & 7 & PP.AM.7\%MMTO & Primária \\
\hline 14 & 78 & 15 & 7 & PP.AM.7\%MMTO.1R & 1 \\
\hline 15 & 78 & 15 & 7 & PP.AM.7\%MMTO.4R & $\mathrm{X}^{*}$ \\
\hline
\end{tabular}

*amostras cujo reprocessamento não foi possível de ser realizado.

As análises de microscopia eletrônica de varredura (MEV- microscópio modelo JSM 6701F) foram realizadas com as amostras fraturadas obtidas logo após o ensaio de tração. Nestas análises, buscou-se observar a dispersão da nanoargila ao longo da fratura dos corpos de prova.

Os ensaios de tração foram realizados de acordo com a norma ASTM D638 ${ }^{[7]}$, em uma máquina Universal de ensaios EMIC modelo DL10000/700 com uma célula de carga de $500 \mathrm{~N}$ e velocidade de 10 $\mathrm{mm} . \mathrm{min}^{-1}$. As análises térmicas de TGA foram realizadas pelo equipamento TGA-Q50/TA Instruments, as amostras com $5,0 \mathrm{mg}$ foram aquecidas de 25 até $1000^{\circ} \mathrm{C} \mathrm{min}{ }^{-1}$ a uma taxa de aquecimento de $10{ }^{\circ} \mathrm{C} \mathrm{min}{ }^{-1}$. As análises de DSC foram realizadas no equipamento DSC-Q20/TA Instruments, onde cerca de 5,0 mg de cada amostra foram aquecidas de 25 a $200{ }^{\circ} \mathrm{C}$ a uma taxa de aquecimento de $10{ }^{\circ} \mathrm{C} \mathrm{min}{ }^{-1}$ (primeiro aquecimento) e mantidas a esta temperatura por dois minutos. Subsequentemente, elas foram resfriadas rapidamente até -90 ${ }^{\circ} \mathrm{C}$ (jumping) e aquecidas novamente até $200{ }^{\circ} \mathrm{C} \mathrm{min}^{-1}$ (segundo aquecimento).

\section{RESULTADOS}

\subsection{Caracterização MEV}

As micrografias de MEV obtidas nos fornecem informações a respeito da morfologia das fases, bem como da dispersão da nanoargila na matriz polimérica. As micrografias das formulações produzidas conforme Tabela 1 estão apresentadas na Figura 1 e Figura 2. 


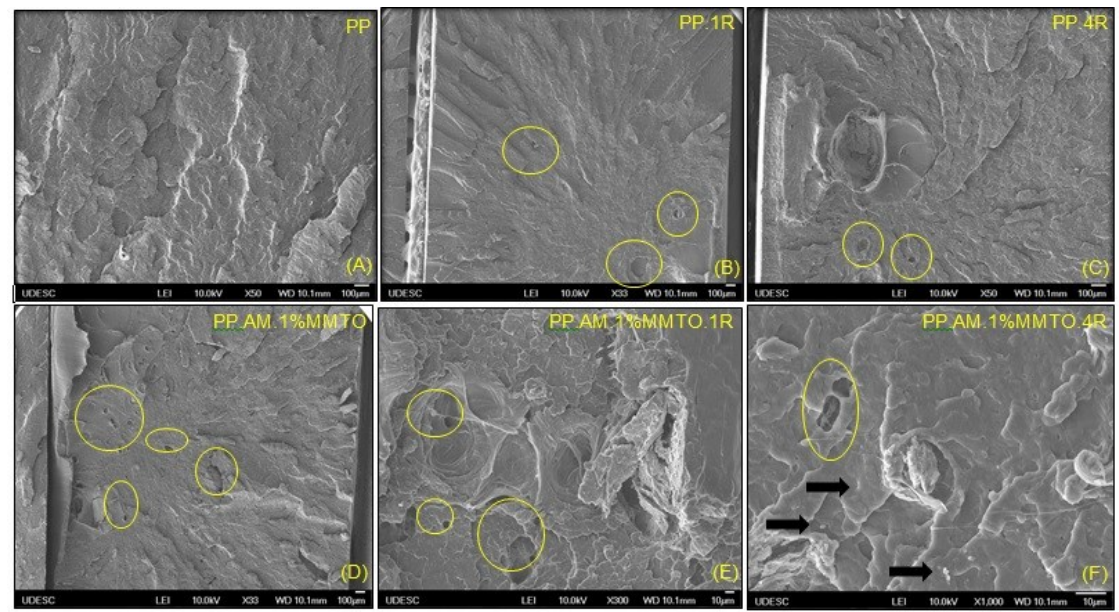

Figura 1: Micrografias de superfície de fratura dos corpos de prova: PP (A), PP.1R (B), PP.4R (C), PP.AM.1\%MMTO (D), PP.AM.1\%MMTO.1R (E) e PP.AM.1\%MMTO.4R (F). É possível identificar alguns vazios, circulados em amarelo nas imagens (B), (C), (D), (E), (F) e as setas indicam a possível presença de aglomerados de nanoargila.

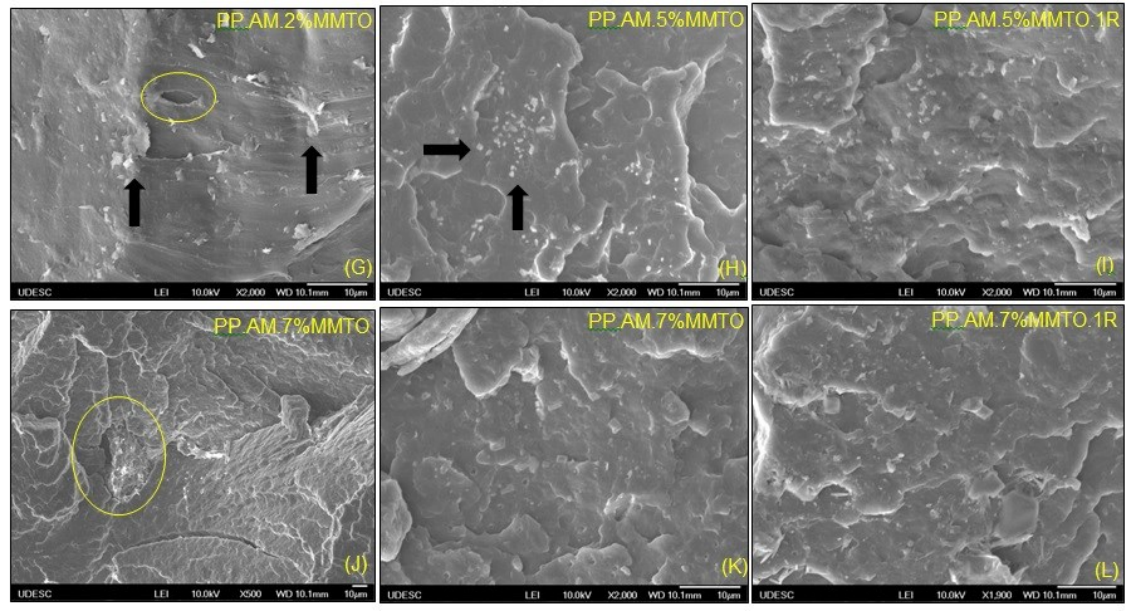

Figura 2: Micrografias de superfície de fratura dos corpos de prova:PP.AM.2\%MMTO (G), PP.AM.2\%MMTO.1R (H), PP.AM.5\%MMTO (I), PP.AM.5\%MMTO.1R (J), PP.AM.7\%MMTO (K) e PP.AM.7\%MMTO.1R (L). É possível identificar alguns vazios, circulados em amarelo nas imagens, $(\mathrm{G})$ e as setas indicam a possível presença de aglomerados de nanoargila.

Em geral todas as micrografias apresentaram uma superfície com fratura frágil. Comparando o PP com as formulações PP.1R e PP.4R notou-se pouca diferença nas superfícies, mas é possível ver o direcionamento dos relevos mais acentuados, partindo do centro para as extremidades e, alguns pontos de remoção de material na imagem (B) e (C) circulados em amarelo. Essa remoção de material pode ter sido ocasionada devido à sua degradação e pela influência nos reprocessos (reciclagem). Os autores CÁCERES E CANEVAROLO ${ }^{[8]}$ obtiveram resultados semelhantes em seus estudos e explicaram que esse fato pode ter ocorrido devido ao aumento de grupos carbonílicos e insaturações

As micrografias da Figura 1 (D), (E) e (F) são formulações contendo 1\% de nanoargila e a imagem (E) é da formulação reprocessada pela primeira vez (PP.AM.1\%MMTO.1R) da forma primária. Foram realizadas varreduras em diferentes pontos da superfície, com diferentes aumentos, onde não foi possível identificar nanoargila ou possíveis pontos que poderiam indicar a presença de nanoargila. Na micrografia (F) referente ao quarto reprocessamento (PP.AM.1\%MMTO.4R) foi possível identificar alguns pontos em branco, que possivelmente podem ser a nanoargila dispersa na matriz polimérica.

Nas micrografias da Figura $2(\mathrm{G})$ e $(\mathrm{H})$, com concentração de 2 e 5\% do MMTO em sua forma primária (PP.AM.2\%MMTO e PP.AM.5\%MMTO) foi possível identificar a presença da nanoargila na forma de pontos mais claros (brancos), indicados por setas. A micrografias da Figura 2 (I) da formulação contendo 5\% 
de nanoargila reprocessada uma vez (PP.AM.5\%MMTO.1R) apresenta resultado semelhante a sua forma primária. Nas micrografias da Figura $2(\mathrm{~J})$ e $(\mathrm{K})$, com concentração de 7\% de MMTO na sua forma primária (PP.AM.7\%MMTO), e na imagem (k) com aproximação de 2000x, identificou-se a presença da nanoargila com maior facilidade devido o seu alto percentual.

Na maioria das micrografias é possível identificar alguns vazios e aglomerados de nanoargila, circulados em amarelo nas imagens (B), (C), (D), (E), (F), (G) e (J), onde BARROS ${ }^{[9]}$ afirma em seu estudo que houve uma má dispersão da nanoargila na matriz polimérica. WASCHBURGUER ${ }^{[10]}$ obteve resultados semelhantes, informando que a má dispersão pode ocorrer devido à mistura da nanoargila ter sido feita em pó e não pelo uso de masterbatch, ocasionando esses vazios e aglomerados de nanoargila. Já LIMA ${ }^{[11]}$ explica que devido a múltiplos reprocessamentos, os aglomerados de nanoargila são quebrados no cisalhamento da rosca da extrusora, sendo assim é possível identificar com mais facilidade a nanoargila na formulação PP.AM.1\%MMTO.4R do que na sua forma primária (PP.AM.1\%MMTO).

\subsection{Resistência à tração}

Os resultados dos ensaios de tração das amostras produzidas conforme as formulações apresentadas na Tabela 1 estão resumidos na Tabela 2 .

Tabela 2: Resultados dos ensaios de tração das formulações PPv, PPv.1R , PPv.4R, PPv.AM, PP.AM.1\%MMTO, PP.AM.1\%MMTO.1R, PP.AM.1\%MMTO.4R， PP.AM.2\%MMTO， PP.AM.5\%MMTO， PP.AM.5\%MMTO.1R, PP.AM.7\%MMTO, PP.AM.7\%MMTO.1R

\begin{tabular}{|l|l|l|l|}
\hline AMOSTRAS & $\begin{array}{l}\text { PRESISTÊNCIA À } \\
\text { TRAÇÃO (MPA) }\end{array}$ & $\begin{array}{l}\text { MÓDULO DE } \\
\text { YOUNG (MPA) }\end{array}$ & $\begin{array}{l}\text { ALONGAMENTO NA } \\
\text { RUPTURA (\%) }\end{array}$ \\
\hline PP & $24,71 \pm 0,3$ & $1042 \pm 15$ & $10,10 \pm 1,9$ \\
\hline PP.1R & $24,52 \pm 0,3$ & $1065 \pm 24$ & $9,37 \pm 0,9$ \\
\hline PP.4R & $24,77 \pm 0,2$ & $1079 \pm 31$ & $9,62 \pm 1,4$ \\
\hline PP.AM.1\%MMTO & $23,88 \pm 0,4$ & $1049 \pm 29$ & $10,31 \pm 0,7$ \\
\hline PP.AM.1\%MMTO.1R & $23,86 \pm 0,3$ & $1044 \pm 36$ & $7,10 \pm 2,2$ \\
\hline PP.AM.1\%MMTO.4R & $24,30 \pm 0,4$ & $1040 \pm 27$ & $9,13 \pm 1,9$ \\
\hline PP.AM.2\%MMTO & $23,67 \pm 0,3$ & $1006 \pm 59$ & $8,93 \pm 1,0$ \\
\hline PP.AM.5\%MMTO & $23,21 \pm 0,5$ & $923,5 \pm 52$ & $6,59 \pm 0,7$ \\
\hline PP.AM.5\%MMTO.1R & $23,71 \pm 0,5$ & $973,8 \pm 53$ & $7,98 \pm 2,0$ \\
\hline PP.AM.7\%MMTO & $22,36 \pm 0,4$ & $935 \pm 42$ & $5,75 \pm 0,6$ \\
\hline PP.AM.7\%MMTO.1R & $23,43 \pm 0,2$ & $972 \pm 59$ & $6,38 \pm 0,5$ \\
\hline
\end{tabular}

De acordo com os resultados contidos na Tabela 2 para as amostras nomeadas de PP, PP.1R e PP.4R, percebe-se que o número de ciclos de reciclagem não afetou de forma significativa a resistência à tração, porém causou um pequeno aumento no módulo de Young, o que indica que o material se tornou ligeiramente mais rígido. Com relação ao alongamento à ruptura, percebe-se que houve uma pequena diminuição desta propriedade com o aumento do número de ciclos de reciclagem, o que representa que a reciclagem do PP levou a uma perda da capacidade do material escoar quando submetido a uma determinada solicitação mecânica. Os valores encontrados de resistência à tração, módulo de Young e alongamento de ruptura, mostram que as variações sofridas estão dentro da margem de erro, permitindo afirmar que não houve perda ou aumento da propriedade.

Analisando os resultados da resistência à tração, do módulo de Young e do alongamento na ruptura mostrados na Tabela 2 para as formulações contendo nanoargila nas suas formas primárias (PP.AM.1\%MMTO, PP.AM.2\%MMTO, PP.AM.5\%MMTO e PP.AM.7\%MMTO) e comparando-os com os resultados do PP, nota-se que houve uma tendência à redução de todas estas propriedades mecânicas com 0 aumento da quantidade de nanoargila na formulação. Ao se avaliar o efeito da reciclagem para as amostras contendo nanoargila (PP.AM.1\%MMTO.1R, PP.AM.5\%MMTO.1R e PP.AM.7\%MMTO.1R), nota-se um pequeno aumento da resistência à tração, módulo de Young e alongamento na ruptura para as formulações contendo $5 \mathrm{e} 7 \%$ de nanoargila em relação às suas formas primárias, ou seja, antes de serem submetidas a um ciclo de reciclagem. Estes resultados sugerem que o fato do material ter sido submetido à reciclagem pode ter contribuído para uma melhor homogeneização do nanoaditivo na matriz polimérica e, desta forma, foi possí- 
vel melhorar as propriedades mecânicas do PP após apenas um ciclo de reciclagem. Para a amostra com 1\% de nanoargila, comparando-se as propriedades mecânicas antes e após um ciclo de reciclagem, observa-se que praticamente não houve influência da reciclagem sobre a resistência à tração e sobre o módulo de Young. Contudo, ao se analisar o alongamento na ruptura, verifica-se uma redução de cerca de $30 \%$ após a amostra ter sido submetida a um ciclo de reciclagem. Quando as propriedades mecânicas das amostras recicladas uma vez são comparadas entre elas, percebe-se que o aumento da quantidade de nanoargila incorporada levou a uma pequena redução da resistência à tração, do módulo de Young e do alongamento na ruptura, assim como verificado para as amostras contendo nanoargila que não foram submetidas ao processo de reciclagem. A redução no módulo de Young e a baixa variação da resistência à tração são explicadas por BOHN ${ }^{[12]}$, onde está atribuída à formação de aglomerados, devido à má dispersão da nanoargila na matriz polimérica.

Por fim, avaliando-se a influência do número de ciclos de reciclagem das amostras contendo $1 \%$ de nanoargila, nota-se que após quatro ciclos de reciclagem o PP tende a melhorar sua resistência à tração e o alongamento na ruptura, enquanto que o módulo de Young diminui muito pouco. A melhora observada na resistência à tração e no alongamento à ruptura após quatro ciclos de reciclagem devem ser uma reflexo de uma melhora na homogeneização e dispersão da nanoargila na matriz polimérica pelo fato do material ter sido fundido e misturado quatro vezes na rosca transportadora da injetora. Dos trabalhos disponíveis na literatura sobre nanocompósitos de matrizes olefínicas (polipropileno), observou-se que a compatibilidade em termos de adesão interfacial polímero/nanoargila ainda é um desafio em aberto, fato comprovado pela pouca influência do argilo-mineral nas propriedades mecânicas destes materiais, assim como na dificuldade para se obter um bom nível de esfoliação do argilo-mineral MORELLI e RUVOLO FILHO ${ }^{[13]}$.

\subsection{Análise termogravimétrica (TGA)}

Os resultados do ensaio de TGA para as amostras produzidas conforme as formulações apresentadas na Tabela 1 estão resumidos na Tabela 3 e as curvas que geraram esses valores estão apresentadas na Figura 3. Devido a grande quantidade de amostras, as curvas termogravimétricas (curvas TG) foram divididas em dois blocos: Figura 3a as formulações que não contém nanoargila e Figura $3 b$ as formulações que contém nanoargila com seus diferentes percentuais aplicados.

Tabela 3: Dados da $\mathrm{T}_{\text {onset }}$ e $\mathrm{T}_{\text {máx }}$ obtidos das curvas de TGA para as amostras PP, PP.1R, PP.4R, PP.AM.1\%MMTO, PP.AM.1\%MMTO.1R, PP.AM.1\%MMTO.4R， PP.AM.2\%MMTO， PP.AM.5\%MMTO， PP.AM.5\%MMTO.1R, PP.AM.7\%MMTO e PP.AM.7\%MMTO.1R

\begin{tabular}{|l|l|l|}
\hline AMOSTRAS & TONSET $\left({ }^{\circ} \mathrm{C}\right)$ & TMÁX $\left({ }^{\circ} \mathrm{C}\right)$ \\
\hline PP & 378,0 & 434,4 \\
\hline PP.1R & 353,9 & 395,9 \\
\hline PP.4R & 368,3 & 420,1 \\
\hline PP.AM.1\%MMTO & 371,5 & 426,3 \\
\hline PP.AM.1\%MMTO.1R & 362,9 & 408,2 \\
\hline PP.AM.1\%MMTO.4R & 363,2 & 412,8 \\
\hline PP.AM.2\%MMTO & 442,6 & 457,7 \\
\hline PP.AM.5\%MMTO & 432,6 & 451,8 \\
\hline PP.AM.5\%MMTO.1R & 365,2 & 412,3 \\
\hline PP.AM.7\%MMTO & 437,4 & 454,4 \\
\hline PP.AM.7\%MMTO.1R & 380,7 & 431,4 \\
\hline
\end{tabular}




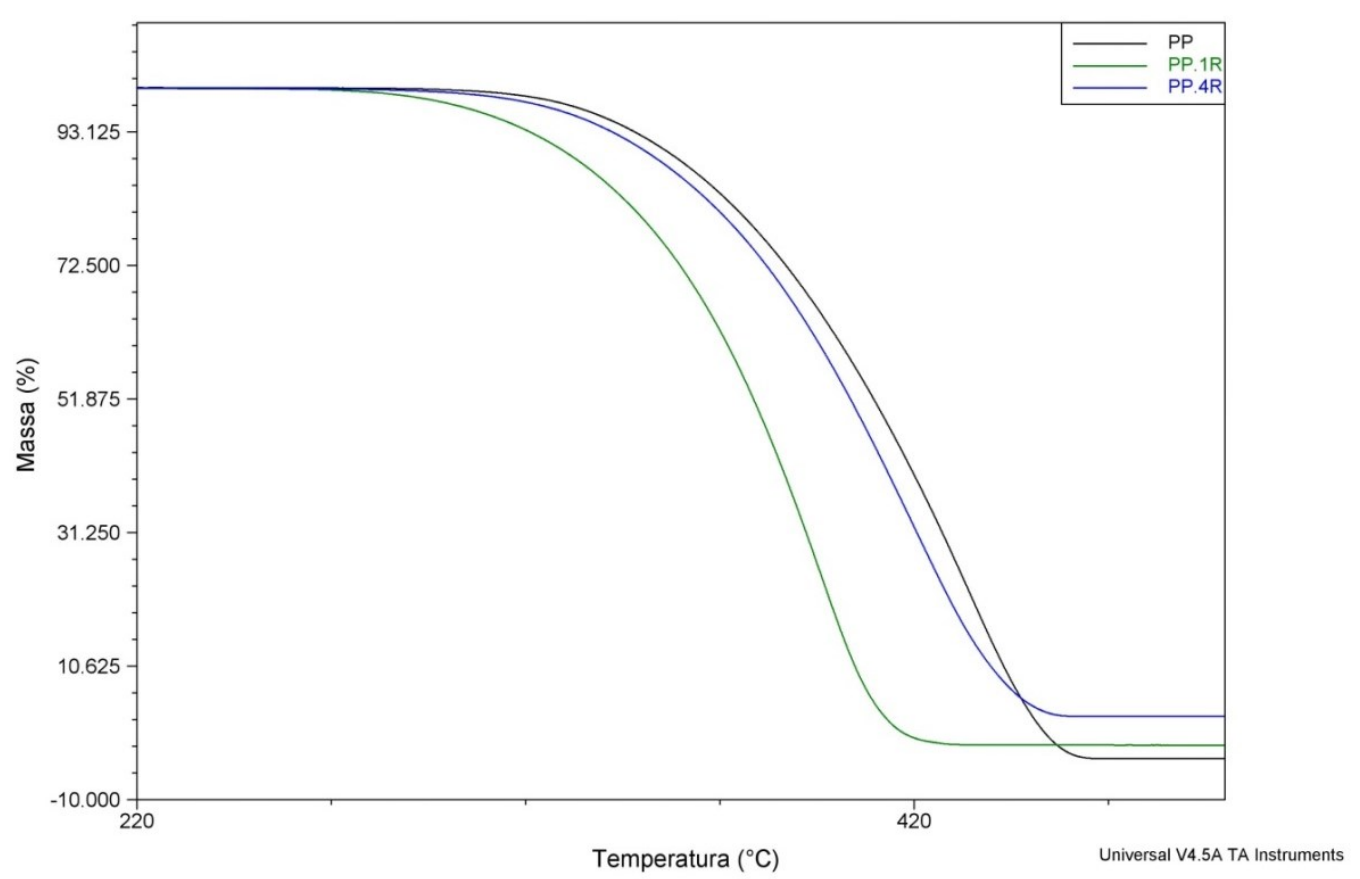

Figura 3a: Curvas de TGA para as formulações: PP, PP.1R e PP.4R.

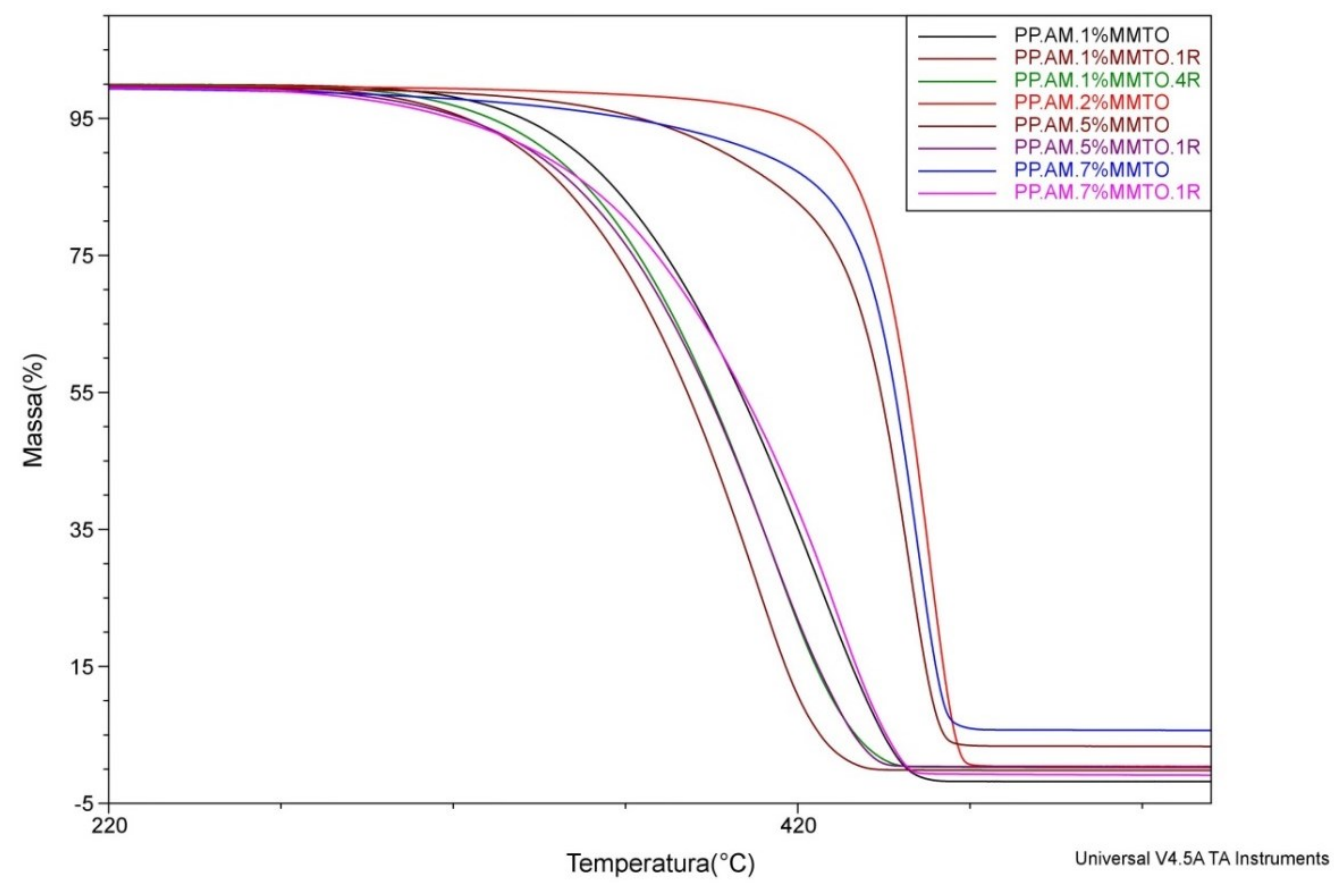

Figura 3b: Curvas de TGA para as formulações: PP.AM.1\%MMTO, PP.AM.1\%MMTO.1R, PP.AM.1\%MMTO.4R, PP.AM.2\%MMTO, PP.AM.5\%MMTO, PP.AM.5\%MMTO.1R, PP.AM.7\%MMTO e PP.AM.7\%MMTO.1R.

As curvas termogravimétricas para todas as formulações demostram apenas um estágio de perda de massa e que aproximadamente $100 \%$ da massa foi consumida. Os resultados mostram que a formulação PP apresentou a $\mathrm{T}_{\text {onset }}$ igual a $378,0^{\circ} \mathrm{C}$ e $\mathrm{T}_{\text {máx }}$ de $434,4^{\circ} \mathrm{C}$. Segundo RABELLO ${ }^{[14]}$ a temperatura de degradação para o PP é de $380,0^{\circ} \mathrm{C}$, muito próxima da encontrada nesse estudo.

Analisando e comparando o PP com as formulações reprocessadas PP.1R e PP.4R (Figura 3a), ficou nítido que o PP é mais estável termicamente, pois as formulações reprocessadas apresentaram uma queda de 24,1 e $9,7^{\circ} \mathrm{C}$ na $\mathrm{T}_{\text {onset, }}$, respectivamente. Essa diminuição na temperatura pode estar relacionada ao processo 
de reciclagem do material (moagem e injeção).

Analisando as formulações contendo nanoargila (Figura 3b), com a adição de $1 \%$ de nanoargila (PP.AM.1\%MMTO) ocorreu uma queda de $6,50{ }^{\circ} \mathrm{C}$ na $\mathrm{T}_{\text {onset }}$ em relação ao PP. Entretanto, a partir da adição de $2 \%$ de nanoargila houve o aumento da resistência térmica para todas as composições em relação ao PP, com destaque para a formulação com concentração de $2 \%$ de nanoargila (PP.AM.2\%MMTO), que obteve um aumento na $\mathrm{T}_{\text {onset }}$ de $64,66^{\circ} \mathrm{C}$ ou $17 \%$. Para BOTELHO et al. ${ }^{[15]}$ esse aumento pode ser atribuído à afinidade da argila organofílica com o polímero, ou seja, uma interação entre as lamelas da argila com a estrutura polimérica. ZEHETMEYER ${ }^{[16]}$ complementa que uma barreira de proteção foi formada pela argila quando migrada para a superfície, bloqueando a liberação de gases a partir da decomposição.

Para as formulações PP.AM.5\%MMTO e PP.AM.7\%MMTO, houve um aumento da estabilidade térmica em relação ao $\mathrm{PP}$ de $\mathrm{T}_{\text {onset }}$ de 54,65 e $59,45^{\circ} \mathrm{C}$ respectivamente, mas uma queda em relação a formulação PP.AM.2\%MMTO. Para PEIXOTO ${ }^{[17]}$ essa diminuição ocorreu por ser empregado elevadas quantidades de MMTO, já que o excesso de carga aumenta a tendência de aglomeração, fato este comprovado pelas imagens realizadas por MEV.

Comparando as formulações reprocessadas (PP.AM.1\%.MMTO.1R, PP.AM.1\%.MMTO.4R, PP.AM.5\%MMTO.1R e PP.AM.7\%MMTO.1R) com suas formulações primárias (PP.AM.1\%MMTO, PP.AM.5\%MMTO e PP.AM.7\%MMTO), os valores da temperatura inicial de degradação são inferiores a $8,54^{\circ} \mathrm{C}, 8,31^{\circ} \mathrm{C}, 67,42^{\circ} \mathrm{C}$ e $56,73^{\circ} \mathrm{C}$, respectivamente, indicando que após o processo mecânico de reprocessamento elas sofrem modificações estruturais que interferem na estabilidade térmica do polímero, apresentando uma diminuição na temperatura de resistência térmica do material. Apenas a formulação PP.AM.7\%MMTO.1R apresentou temperatura de degradação superior ao $\mathrm{PP}$, em torno de $2,72^{\circ} \mathrm{C}$. As demais amostras reprocessadas apresentaram valores inferiores. Para DINTCHEVA ${ }^{[18]}$, de modo geral o reprocessamento (reciclagem) por se tratar de um processo de fusão pode originar degradação oxidativa e termomecânica do polímero, resultando na modificação da estrutura molecular do polímero e ocasionando uma deterioração subsequente das propriedades mecânicas, térmicas e ópticas, alterando a taxa de transmissão de gás, resultando na menor estabilidade térmica.

\subsection{Calorimetria exploratória diferencial (DSC)}

Os resultados do ensaio de DSC para as amostras produzidas conforme as formulações apresentadas na Tabela 1 estão resumidos na Tabela 4 e as curvas que geraram esses valores estão apresentadas na Figura 4. A Figura 4a apresenta as curvas de DSC para segundo aquecimento para a formulação de PP e suas formas recicladas e a Figura $4 \mathrm{~b}$ apresenta as curvas de DSC para as formulações contendo nanoargila na forma primária e suas formulações reprocessadas.

a)

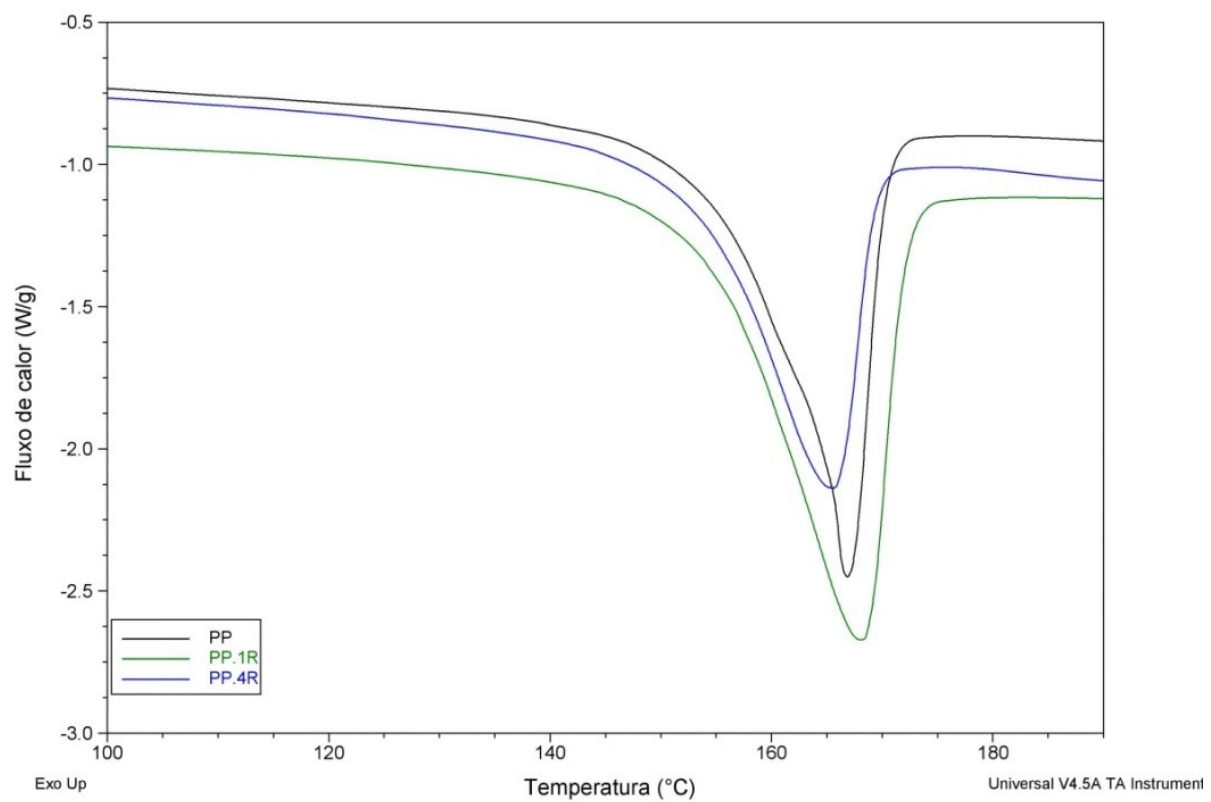

Figura 4a: Curvas de DSC para as formulações: PP, PP.1R e PP.4R 


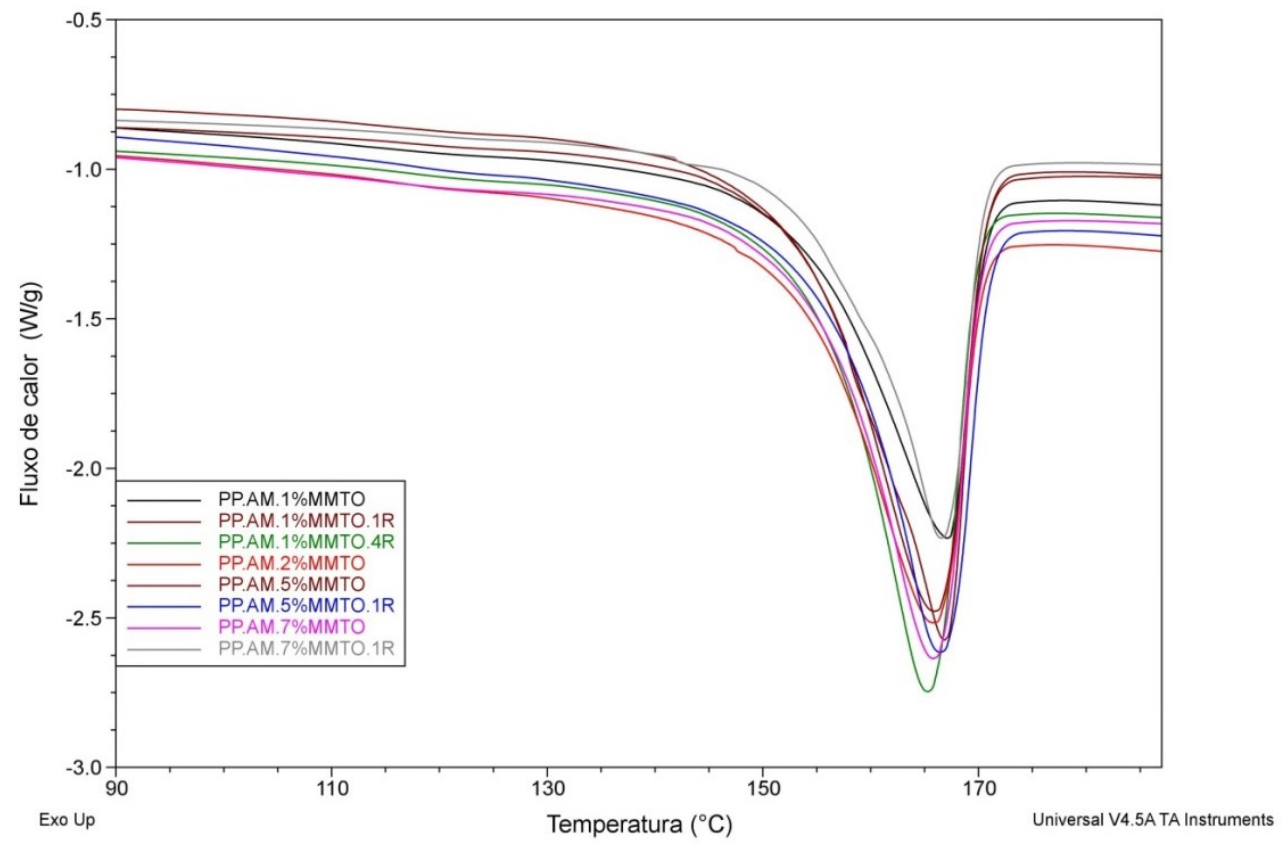

Figura 4b: Curvas de DSC para as formulações: PP.AM.1\%MMTO, PP.AM.1\%MMTO.1R, PP.AM.1\%MMTO.4R, PP.AM.2\%MMTO, PP.AM.5\%MMTO, PP.AM.5\%MMTO.1R, PP.AM.7\%MMTO e PP.AM.7\%MMTO.1R

A Tabela 4 apresenta os resultados referentes à $\mathrm{T}_{\mathrm{m} 2}\left({ }^{\circ} \mathrm{C}\right), \mathrm{T}_{\mathrm{C}}\left({ }^{\circ} \mathrm{C}\right), \Delta \mathrm{Hm}(\mathrm{J} / \mathrm{g})$ e $\alpha \mathrm{c}(\%)$, durante o aquecimento aos quais foram submetidas às amostras de nanocompósitos.

Tabela 4: Dados da Tonset e Tmáx obtidos das curvas de TGA para as amostras PP, PP.1R, PP.4R, PP.AM.1\%MMTO, PP.AM.1\%MMTO.1R, PP.AM.1\%MMTO.4R， PP.AM.2\%MMTO， PP.AM.5\%MMTO， PP.AM.5\%MMTO.1R, PP.AM.7\%MMTO e PP.AM.7\%MMTO.1R

\begin{tabular}{|l|l|l|c|c|}
\hline AMOSTRAS & TM2 $\left({ }^{\circ} \mathbf{C}\right)$ & TC $\left({ }^{\circ} \mathbf{C}\right)$ & $\Delta$ HM (J/G) & AC (\%) \\
\hline PP & 166,9 & 101,0 & 88,1 & 53,3 \\
\hline PP.1R & 168,1 & 101,2 & 109,0 & 65,9 \\
\hline PP.4R & 165,5 & 103,6 & 76,4 & 46,2 \\
\hline PP.AM.1\%MMTO & 167,1 & 98,6 & $76,3^{*}$ & 46,2 \\
\hline PP.AM.1\%MMTO.1R & 166,9 & 99,9 & $101,7^{*}$ & 61,5 \\
\hline PP.AM.1\%MMTO.4R & 165,3 & 98,9 & $101,1^{*}$ & 61,2 \\
\hline PP.AM.2\%MMTO & 165,7 & 98,8 & $90,6^{*}$ & 54,8 \\
\hline PP.AM.5\%MMTO & 165,9 & 99,8 & $110,3^{*}$ & 66,7 \\
\hline PP.AM.5\%MMTO.1R & 166,4 & 100,0 & $95,7^{*}$ & 57,9 \\
\hline PP.AM.7\%MMTO & 165,8 & 99,1 & $102,2^{*}$ & 61,8 \\
\hline PP.AM.7\%MMTO.1R & 166,5 & 102,3 & $82,8^{*}$ & 50,1 \\
\hline
\end{tabular}

$* \Delta \mathrm{Hm}(\mathrm{J} / \mathrm{g})$ - Corrigido para a fração mássica da nanoargila

Em todas as curvas pode-se observar a presença de apenas um pico endotérmico para todas as formulações analisadas. Verificou-se com auxílio dos valores da Tabela 4 que a $\mathrm{T}_{\mathrm{m} 2}$ não apresentou alterações significativas, contendo ou não percentuais de MMTO, sendo primária ou reprocessada, quando comparadas ao PP. A presença da nanoargila parece não interferir nas forças intermoleculares das cadeias do polímero. A 
temperatura de fusão para o PP encontrada foi de $166,9{ }^{\circ} \mathrm{C}$, valor esse em acordo com o que é sugerido pela literatura de MANO ${ }^{[19]}$, ZEHETMEYER ${ }^{[16]}$ e com o valor fornecido pelo fabricando, sendo esses valores $165,0{ }^{\circ} \mathrm{C}, 169,0{ }^{\circ} \mathrm{C}$ e $164,0{ }^{\circ} \mathrm{C}$, respectivamente.

Analisando a temperatura de cristalização do PP e comparando com as formulações PP.1R e PP.4R, obteve-se um aumento de 0,20 e $2,60{ }^{\circ} \mathrm{C}$ respectivamente, valores esses dentro da margem dos erros experimentais, mostrando que a $\mathrm{T}_{\mathrm{c}}$ não sofreu variações significativas.

Quando comparado o PP com as formulações contendo nanoargila na forma primária ou reprocessada (PP.AM.1\%MMTO, PP.AM.1\%MMTO.1R, PP.AM.1\%MMTO.4R, PP.AM.2\%MMTO, PP.AM.5\%MMTO, PP.AM.5\%MMTO.1R, PP.AM.7\%MMTO e PP.AM.7\%MMTO.1R), os resultados obtidos foram -2,40, $1,10,-2,10,-2,20,-1,20,-1,00,-1,90$ e $1,30{ }^{\circ} \mathrm{C}$ respectivamente. Foi possível observar que não houve variação significativa também na temperatura de cristalização, quando adicionado os percentuais de nanoargila para todas as formulações citadas anteriormente, sendo primária ou reprocessada. Para WASCHBURGUER [10], a nanoargila não apresentou efeito de nucleação na cristalização do PP, devido à má dispersão na matriz polimérica, podendo ser atribuído ao equipamento usado no processamento. Fato esse comprovado por MEV, onde foram encontrados aglomerados de nanoargila.

A $\mathrm{T}_{\mathrm{c}}$ para o PP foi de $101,09{ }^{\circ} \mathrm{C}$ e está em acordo com que o fabricante indica que é de $102,0{ }^{\circ} \mathrm{C}$. Analisando o grau de cristalinidade do PP e comparando com as formulações contendo nanoargila na forma primária, a amostra com 1\% (PP.AM.1\%MMTO) apresentou uma queda de 53,30 para 46,20\% em sua cristalinidade, no entanto, quando adicionado $2 \%$ (PP.AM.2\%MMTO), a cristalinidade aumentou de 53,30 para 54,8 \% em relação ao PP e de 46,20 para 54,80 \% em relação a formulação PP.AM.1\%MMTO. O melhor resultado obtido de cristalinidade foi com a formulação PP.AM.5\%MMTO, onde seu $\alpha$ c foi de $66,70 \%$. A amostra contendo 7 \% (PP.AM.7\%MMTO), de nanoargila possui a cristalinidade de 61,80\% maior que o PP que é de 53,3 \%, porém menor que a amostra contendo $5 \%$ (PP.AM.5\%MMTO).

Com relação às formulações reprocessadas PP.1R, PP.AM.1\%MMTO.1R, PP.AM.1\%MMTO.4R e PP.AM.5\%MMTO.1R, o grau de cristalinidade foi de 65,90, 61,50, 61,20 e 57,90\%, respectivamente. Os valores obtidos são superiores a suas formas primárias e também em relação ao PP. Para as duas formulações PP.AM.7\%MMTO.1R e PP.4R, o grau de cristalinidade foi inferior com relação ao PP, onde foram de 50,10 e 46,20\% e também a suas formas primárias. O aumento da cristalinidade para os materiais reprocessados pode ter sido causado pela cisão molar durante a reciclagem (moagem e injeção), o que provoca uma redução da massa molecular, aumentando a mobilidade das cadeias poliméricas que podem se orientar e aumentar a predisposição para cristalinização ${ }^{[20]}$.

\section{CONCLUSÕES}

As micrografias das amostras da superfície realizadas por MEV para os nanocompósitos obtidos revelaram que não houve uma boa dispersão da nanoargila na matriz polimérica de PP, resultando em aglomerados observados nas superfícies das fraturas analisadas que, em geral, apresentaram-se frágeis. Encontrou-se nanoargila a partir de formulações contendo $1 \%$. Devido aos aumentos de escala do microscópio ser na ordem de micrometros, não foi possível definir o tipo de estrutura obtida (intercalada ou esfoliada).

Por meio de ensaios mecânicos de tração, observou-se que as formulações de PP não apresentaram alterações nas propriedades de resistência a tração, módulo de Young e alongamento de ruptura quando foram reprocessadas até o quarto ciclo, demostrando que a reciclagem de $100 \%$ do material poderá garantir produtos finais de qualidade. A adição de nanoargila não apresentou ganhos nas propriedades mecânicas analisadas, pois os valores de resistência a tração $(24,71 \pm 0,28 \mathrm{MPa})$, módulo de Young $(1042 \pm 15,18 \mathrm{Mpa})$ e alongamento de ruptura $(10,10 \pm 1,84 \%)$ do PP reduziram gradativamente com o aumento do percentual da MMTO $(1,2,5$ e $7 \%)$.

As análises de TGA mostraram que o processo de reciclagem do PP diminui a estabilidade térmica do material. A incorporação de nanoargila a partir de $2 \%$ elevou consideravelmente a estabilidade térmica das formulações se comparado ao PP, sendo a formulação contendo $2 \%$ de MMTO a que apresentou melhor resultado, elevando a estabilidade térmica em $64,66^{\circ} \mathrm{C}$. Para as formulações reprocessadas contendo nanoargila, os resultados são inferiores as suas formas primárias, mas superiores ainda ao PP.

Por meio de DSC verificou-se que para todas as formulações estudadas não houve modificação da temperatura de fusão.

Os resultados de resistência à tração e alongamento de ruptura encontrados nesse trabalho obtiveram resultados semelhantes aos estudos que utilizaram os materiais em forma de masterbatch e extrusora de rosca dupla para o processamento. As analises térmicas indicaram um aumento na estabilidade térmica do material 
e um aumento no grau de cristalização para maioria das formulações contendo nanoargila, reprocessadas ou não, mostrando que o método utilizado não afetou essas propriedades. Como melhor resultado obtido analisando as propriedades de todas as formulações, a PP.AM.2\%MMTO, apresentando 2,80 \% de aumento no grau de cristalinidade e $16,93 \%$ de aumento na temperatura de fusão.

Portanto recomenda-se a adição da nanoargila para aplicações especificas, onde necessitam de um material com resistência térmica maior do que o material convencional ou com maior cristalinidade.

\section{AGRADECIMENTOS}

À CAPES, à empresa Spectrochem Ltda pela doação da nanoargila, à empresa Britânia eletrodomésticos Ltda pelo tempo de maquina cedido, à UNIVILLE e à UDESC pelo apoio ao projeto com infraestrutura laboratorial para realização dos ensaios.

\section{BIBLIOGRAFIA}

[1] OLIVEIRA JÚNIOR, A. R. Obtenção de nanocompósitos polipropileno argila compatibilizados com organossilanos. Tese D.Sc., Universidade Estadual de Campinas, Instituto de Química, UNICAMP, Campinas, SP, Brasil, 2006.

[2] BOESEL, L. F. Nanocompósitos de Poli (teraftalato de etileno) e Argila. Dissertação M.Sc., Universidade Federal de São Carlos, São Paulo, SP, Brasil, 2001.

[3] PAIVA, L. B., MORALES, A. R., DIAZ, F. R. V. "Argilas organofílicas: características, metodologias de preparação, compostos de intercalação e técnicas de caracterização", Cerâmica, v.54, n.330, pp. 213-226, 2008.

[4] VOSSEN, C. A. Nanocompósitos de ABS/PA e argilas organofilicas. Dissertação de M.Sc., Escola Politécnica da Universidade de São Paulo, São Paulo, SP, Brasil, 2009.

[5] ARDANUY, M. et al. "Non-isothermal crystallization kinetics and activity of filler in polypropylene/mgal layered double hydroxide nanocomposites", Thermochimica Acta, v. 479, pp. 45-52, 2008.

[6] SANTOS, F. A. Estudo de misturas de HDPE e serragem de cedro para obtenção de madeira plástica. 2008. Dissertação de M.Sc, Universidade Federal do Rio de Janeiro (UFRJ), Rio de Janeiro, RJ, Brasil, 2008.

[7] ASTM D-638. "Standard Practice for Conditioning Plastics for Testing", 2008.

[8] CÁCERES, C. A., CANEVAROLO, S. V. "Degradação do polipropileno durante a extrusão e a geração de compostos orgânicos voláteis", Revista Polímeros: Ciência e Tecnologia, v. 19, n.1, pp. 79-84, 2009.

[9] BARROS, N. G. Propriedades do material polimérico obtido da reciclagem de embalagens multicamadas. 2013. Dissertação de M.Sc., Universidade Estadual de Campinas, Campinas, São Paulo, SP, Brasil, 2013.

[10] WASCHBURGUER, M. R. Compósito de polipropileno com nanocarga. Dissertação de M.Sc., Universidade Federal do Rio Grande do Sul (UFGRS), Porto Alegre, RS, Brasil, 2006.

[11] LIMA, L. S. A influência das variáveis de processamento e de diferentes tipos de nanocargas nas propriedades dos compósitos de polipropileno. Dissertação de M.Sc., Universidade de Caxias do Sul, Caxias do Sul, 2015.

[12] BOHN, S. Influência da adição de plastificantes na dispersão de nanoargila em matriz poli (cloreto de vinila). Dissertação M.Sc., Universidade do Estado de Santa Catarina, Joinville, SC, Brasil, 2015.

[13] MORELLI, F. C., RUVOLO FILHO, A. "Nanocompósitos de polipropileno e argila organofílica: difração de raio x, espectroscopia de absorção na região do infravermelho e permeação ao vapor d'água", Polímeros, v. 20, n. 2, pp. 121-125, 2010.

[14] RABELLO, M. S. Aditivos de Polímeros. São Paulo, Artliber, 2000.

[15] BOTELHO, K.T. et al. "Estudo das propriedades térmicas e mecânicas de nanocompósitos, formados a partir de argilas organofílicas e polímeros biodegradáveis", In: $55^{\circ}$ Congresso Brasileiro de Cerâmica, Porto de Galinhas, PE, 2011.

[16] ZEHETMEYER, G. Nanocompósitos de polipropileno/montmorilonita: avaliação das propriedades e sua aplicação como embalagens de alimentos. 2012. Dissertação de M.Sc - Universidade Federal do Rio Grande do Sul (UFRGS), Porto Alegre, RS, Brasil 2012.

[17] PEIXOTO, L. S. Preparação de compósitos e nanocompósitos poliméricos in situ. Tese de D.Sc., Universidade Federal do Rio de Janeiro (UFRJ), Rio de Janeiro, RJ, Brasil, 2012. 
[18] DINTCHEVA, N. T. et al. "Recycling of plastics from packaging", Polymer Degradation and Stability, v.57, pp. 191-203, 1997.

[19] MANO, E. B. Introdução a Polímeros. São Paulo, Edgard Blücher, 2004.

[20] MARTINS, M. H., DE PAOLI, M. A. "Polypropylene compounding with post-consumer material: II. Reprocessing". Polymer Degradation and Stability, v.78, pp. 491-495, 2002. 